\title{
Compact Harmonic Rejection Filter for C-band High-Power Satellite Applications
}

\author{
F. Teberio ${ }^{1,2}$, P. Martin-Iglesias ${ }^{3}$, I. Arregui ${ }^{1}$, I. Arnedo ${ }^{1}$, T. Lopetegi ${ }^{1}$, and M. A. G. Laso ${ }^{1}$ \\ ${ }^{1}$ Electrical and Electronic Engineering Department, Public University of Navarre, Pamplona, Spain \\ ${ }^{2}$ Anteral S.L., Pamplona, Spain \\ ${ }^{3}$ ESA/ESTEC, Noordwijk, The Netherlands
}

\begin{abstract}
A compact high-power low-pass filter for C-band broadband satellite applications is presented in this paper. The filter is composed of three different sections. $A 9^{\text {th }}$-order compact high-power multi-ridge structure achieves the fundamental mode stopband and the suppression of all-higher order modes. The required slope between the pass- and the stopband is accomplished by means of two step-shaped bandstop elements separated by very short waveguide sections. The passband of the filter is achieved through two compact matching networks. The filter is only 164$\mathrm{mm}$ long, has less than $0.05 \mathrm{~dB}$ of insertion loss, handles $9.6 \mathrm{~kW}$ (single-carrier multipactor analysis), and has a very wide stopband (up to Ku-band). A dramatic size reduction has been achieved with respect to other commercially available solutions.

Keywords - compact filter, harmonic filter, high-power filter, low-pass filter, microwave filter.
\end{abstract}

\section{INTRODUCTION}

In satellite communications, waveguide low-pass filters are employed in the transmission RF chain to reject the spurious harmonics arising from the non-linear operation of the power amplifiers [1]. A channelized approach can be used, where each channel has its own low-pass filter that supports just the power of the channel. However, this is not optimum in terms of mass and volume. To surpass this limitation, a single output low-pass filter can be utilized, provided that it supports the power of all the channels once combined.

Current solutions for C-band output low-pass filters in waveguide technology are based on classical filters such as corrugated and waffle-iron filters. A degradation over the stopband of a corrugated filter can occur due to the propagation of the higher-order $\mathrm{TE}_{\mathrm{n} 0}$ modes, and this impedes achieving harmonic rejection up to the third/fourth harmonic [1]. This is why the waffle-iron filter is a widely-employed solution, as it also achieves the rejection of the higher-order modes in the stopband [2]. However, it is not possible to have a very wide stopband and high-power behavior simultaneously in a single structure. Some high-power improvements have been reported in [3], [4], but they are still limited if a modern C-band satellite application is considered. One of the possible solutions employed by the space industry is to concatenate both a corrugated filter and a waffle-iron filter with large gaps (in order to withstand the power requirements), relaxing the frequency demands of each filter but designed in such a way that, together, they accomplish the required rejection when they are cascaded. As the corrugated filter is not able to reject correctly the harmonics, it is used to achieve the required slope between the pass-band and the stopband and the attenuation up to the cut-off frequency of the $\mathrm{TE}_{20}$ mode. On the other hand, the waffle-iron filter is used to cut the harmonics propagating above the $\mathrm{TE}_{20}$ mode cut-off frequency. Consequently, finding the optimum design for each filter (in such a way that both filters handle also the maximum power) implies finding the optimum $\mathrm{TE}_{20}$-mode cutoff frequency, design each filter separately, and then cascade them together. The design of this combined structure is cumbersome and time-consuming, and the final structure is rather bulky and spurious effects may appear due to concatenation.

Recently, some techniques have been published for overcoming the previous issues of classical designs. In [5], a two-dimensional (2D) multi-ridge structure based on sinusoidal bandstop elements was used to achieve the suppression of the higher-order $\mathrm{TE}_{\mathrm{n} 0}$ modes and a high-power behavior. This technique requires two long windowed sections to achieve the matching of the filter, which increases the final size and insertion loss of the filter. On the other hand, machining nonstep-shaped profiles is rather time-consuming and costly. In [6], a design method for classical waffle-iron filters was proposed, which also allowed responses with multiple transmission zeros. The method rests in alternating waffle-iron and rectangularwaveguide sections, and it is based on controlling the magnitude and phase of the local reflection coefficient in pairs. Using [6], the power-handling of the waffle-iron filter can be improved, but only up to a certain extend. Now, we report on a new (waffle-iron-like) 2D structure, which is composed of stubs of around quarter-wave height that can be placed closely packed, allowing for higher power and compactness. The proposal surpasses [5] in manufacturability and length, and [6] in power-handling and length.

In this paper, a very compact high-power low-pass filter is proposed for harmonic rejection in C-band satellite payloads. Its structure can be divided in three different sections: 1) a 2D structure composed of quarter-wave stubs, which achieves the required stopband of the filter as well as the higher-order mode suppression, 2) several step-shaped bandstop elements to obtain the required slope between the passband and the rejected band, and 3) very short input/output networks based on a few rectangular steps whose heights are optimized to accomplish the return loss and the matching of the filter with the ports

(C) 2020 IEEE. Personal use of this material is permitted. Permission from IEEE must be obtained for all other uses, in any current or future media, including reprinting/republishing this material for advertising or promotional purposes, creating new collective works, for resale or redistribution to servers or lists, or reuse of any copyrighted component of this work in other work. 
dimensions. The solution will benefit from high-power handling capability, since the method allows for large minimum mechanical gaps (actually, this is a free parameter), as well as easy-to-fabricate rectangular profiles. A very reduced size will be also obtained since the bandstop elements can be put closely together as well as their length can be shortened in the propagation direction, as both are also free parameters. A prototype has been manufactured and measured in C-band showing a very good agreement with the simulation results and compared to other commercially available alternatives.

\section{DESIGN PROCEDURE}

The filter presented in this paper is composed of three subsections which are clearly differentiated (see Fig 1). Firstly, the 2D arrangement of bandstop elements is designed. This part achieves the harmonic rejection for the fundamental $\mathrm{TE}_{10}$ mode and the suppression of all higher-order modes. Then, the desired slope between the passband and the stopband is obtained by means of a few step-shaped bandstop elements separated by very short waveguide sections. Finally, two matching networks are added to the input/output ports to accomplish the required return loss and reach the standard port dimensions (see Fig. 1(a)).

\section{A. Fundamental Mode and Higher-Order Mode Rejection}

As in [6], the 2D arrangement of bandstop elements in this paper is also composed of alternating waffle-iron-like and rectangular-waveguide sections. However, in this work we consider quarter-wave height stubs. The design process begins by fixing the height of the highest $\left(h_{w, \max }\right)$ and shortest $\left(h_{w, \min }\right)$ stubs that will reject the minimum and maximum frequency of the stopband of the filter, respectively (Fig 1. (b)). In general, for the stopband frequencies, the height, $h_{w}$, of each rectangular waveguide section and the height of the teeth of the consecutive waffle-iron-like section is around one quarterwave. The length of the rectangular waveguide sections, $l_{w}$, is a free parameter and is defined short enough to minimize size and wide enough to ensure an easy manufacturing by classical milling. Moreover, the stubs can be placed closely together $\left(l_{t}\right)$ since this is also a free parameter. Both design strategies highly reduce the filter length, a particularly interesting feature for $\mathrm{C}$ band.

The design procedure continues by setting several other physical parameters of the bandstop elements in the 2D arrangement, similarly to classical waffle filters and typical well-known considerations [2]. Specifically, the number of teeth, $n_{t}$, (which will be higher for wider stopbands), the length of the teeth, $l_{t}$, their width, $w_{t}$, and their separation, $s_{t}$, which will be fixed equal (or similar) and large enough to allow, at once, an easy fabrication and a good isotropy, providing the same characteristics at a certain frequency, for the TEM waves propagating in any direction (see Fig. 1(b) and Fig. 1 (c)). Furthermore, the 2D arrangement of bandstop elements is defined symmetric in the E-plane. Finally, the minimum mechanical gap, $g_{w}$, is another free parameter and is defined high enough to achieve a high-power behaviour, and short

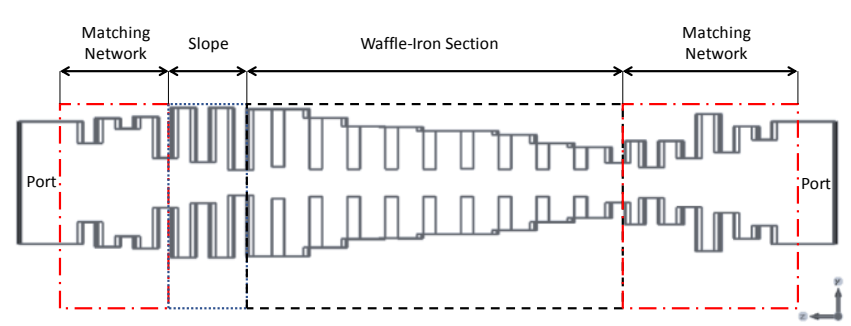

(a)

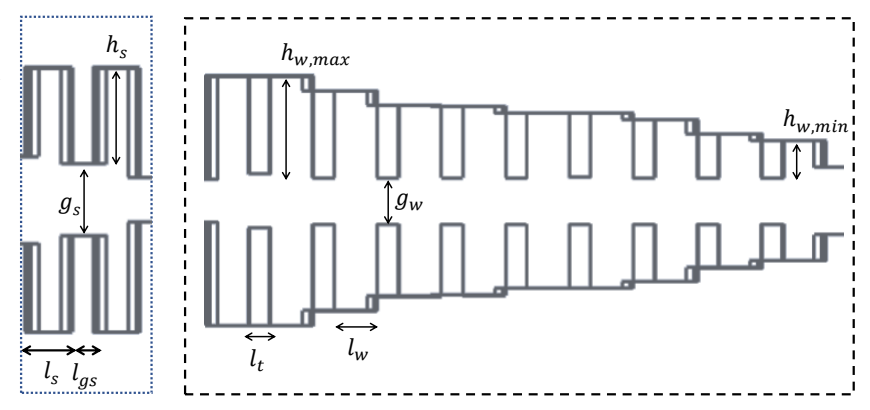

(b)

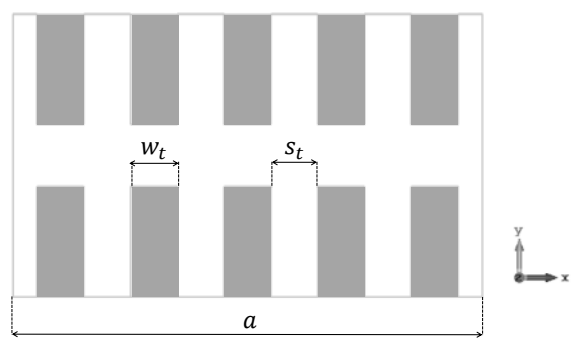

(c)

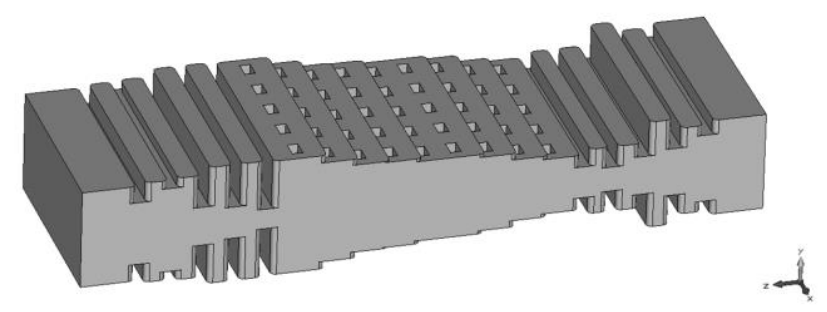

(d)

Fig. 1. Sketch of the novel high-power compact harmonic rejection filter. (a) Side view with the different section of the filter, (b) 2D arrangement of bandstop elements along with its design parameters, (c) transversal section of the 2D arrangement of bandstop elements, and (d) 3-D view of the final structure including the rounding of the corners.

enough to shift the cut-off frequency of all higher order non$\mathrm{TE}_{\mathrm{n} 0}$ modes up to the desired frequency.

\section{B. Slope Between the Pass- and the Rejected Band}

One of the most interesting advantages of this technique is that the required slope between the pass- and the stopband is accomplished by adding a few step-shaped bandstop elements of height $h_{s}$ and length $l_{s}$. It allows a substantial size reduction in comparison with [5] and [6], in which a larger number of sections would be necessary to achieve the same slope. The height and length of the waveguide sections between them will be also defined equal to $g_{s}$ and $l_{g s}$. 


\section{Return Loss and Matching}

The return loss and the matching of the filter is achieved following the technique well described in [7]. With a few optimized-height rectangular steps at the input and the output port of the filter, the required in-band return loss and the standard ports heights are easily reached. The 3D-view of the final structure is shown in Fig. 1(d), which also includes the rounding of the corners that are necessary for manufacturing using milling.

\section{C-BAND FILTER DESIGN AND RESULTS}

According to the design technique presented above, a very compact high-power filter for harmonic rejection for C-band satellite payloads is designed featuring the specifications given in Table I. The height of the largest and shortest bandstop elements is $h_{w, \max }=14.5 \mathrm{~mm}$ and $h_{w, \min }=5.4 \mathrm{~mm}$ in order to reject the minimum and the maximum frequency of the intended stopband, respectively. In order to accomplish the required attenuation, 9 sections of quarter-wave stubs have been used. The length of the rectangular waveguide sections, $l_{w}$ and $l_{s}$, has been fixed to $6 \mathrm{~mm}$ and the length between the step-shaped bandstop elements, $l_{t}$ and $l_{g s}$, equal to $3 \mathrm{~mm}$. After that, the physical dimensions of the filter $n_{t}=5, w_{t}=5.8 \mathrm{~mm}$, and $s_{t}=5.834 \mathrm{~mm}$ are chosen for the filter to have a good isotropy providing the same features (at a given frequency) for all TEM waves propagating in any direction. This achieves the suppression of the higher-order TEn0 modes, minimizes the excitation of the higher-order modes in the inner part of the device, and allows for an easy manufacturability. The gap of the structure has been fixed higher than $g_{w}=g_{s}=6 \mathrm{~mm}$ to allow a high-power behavior.

Two step-shaped bandstop elements of the highest height have been used to achieve the required slope between the passand the stopband $\left(h_{s}=14.82 \mathrm{~mm}\right)$. Additionally, a $4^{\text {th }}$-order and a $2^{\text {nd }}$-order input/output matching networks have been utilized to reach the standard port height and to fulfil the required return loss of the filter. The total length of the filter is only $164 \mathrm{~mm}$. This can be compared to other commercially available alternatives, such as the one developed in a previous ARTES project [8] where, by simple visual inspection, the filter length is around $500 \mathrm{~mm}$.

The fundamental $\mathrm{TE}_{10}$-mode frequency response of the novel filter is shown in Fig. 2 and the higher-order mode suppression is shown in Fig. 3.

The filter has been fabricated by milling in two halves cut by the H-plane using a machining radius equal to $1.5 \mathrm{~mm}$ (see Fig. 4). The prototype has been measured in the WR229, WR159, WR112, WR90 and WR75 bands using proper waveguide-coax transitions and waveguide tapers between WR229 and each band port standard. As it can be seen, the measurements of the prototype (Fig. 5) show an excellent level of attenuation in the stopband of the filter. The return loss is kept below $23 \mathrm{~dB}$ for the passband. The measured insertion loss is better than $0.05 \mathrm{~dB}$ (see Fig 6).

The multipactor threshold of the structure has been estimated using SPARK3D, using the electromagnetic fields previously calculated with CST MWS, at $4.2 \mathrm{GHz}$. The secondary emission yield of ECSS silver has been considered. The input power threshold obtained is better than $9.6 \mathrm{~kW}$ under single carrier continuous wave excitation. Considering the application as output filter and the specifications provided in Table I, a multicarrier analysis is more adequate. A multipactor

\section{TABLE I SUMMARY OF SPECIFICATIONS}

\begin{tabular}{lcc}
\hline \hline Passband & \multicolumn{2}{c}{$3.4-4.2 \mathrm{GHz}$} \\
Return loss & \multicolumn{2}{c}{$>23 \mathrm{~dB}$} \\
Insertion loss & Band $(\mathrm{GHz})$ & Attenuation \\
\hline \hline Rejection bands & $5.0-5.3$ & $30 \mathrm{~dB}$ \\
Rejection band 1 & $5.3-5.8$ & $50 \mathrm{~dB}$ \\
Rejection band 2 & $5.8-6.5$ & $80 \mathrm{~dB}$ \\
Rejection band 3 & $7.0-8.5$ & $80 \mathrm{~dB}$ \\
Rejection band 4 & $10.5-12.7$ & $50 \mathrm{~dB}$ \\
Rejection band 5 & $13.75-14.5$ & $30 \mathrm{~dB}$ \\
Rejection band 5 & \multicolumn{2}{c}{ WR229 } \\
\hline Interface port & \multicolumn{2}{c}{$120 \mathrm{~W}$ x 6 carriers } \\
\hline \hline
\end{tabular}

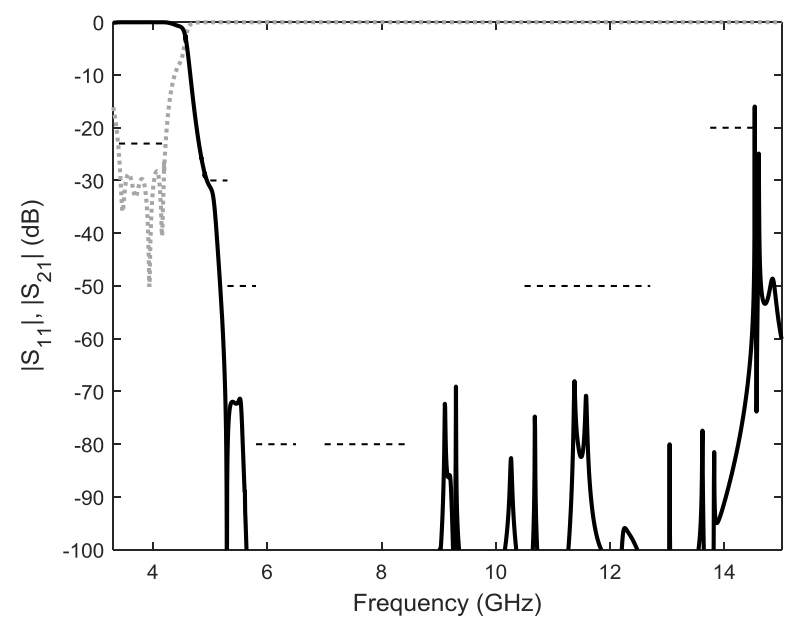

Fig. 2. FEST3D simulation results of the novel filter (black line) when is excited with the fundamental $\mathrm{TE}_{10}$ mode. $\left|S_{11}\right|$ : dotted line; $\left|S_{21}\right|$ : solid line. Frequency mask for return loss and out-of-band rejection with dashed line.

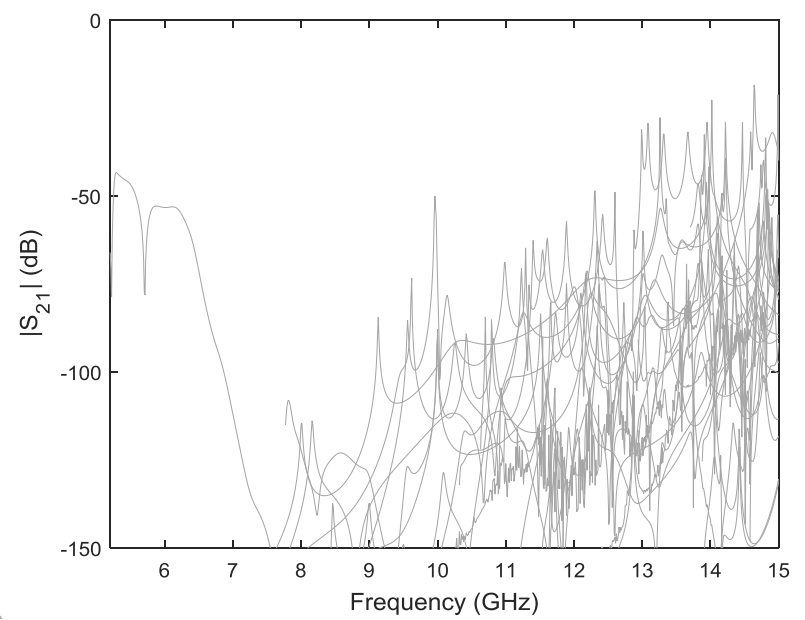

Fig. 3. FEST3D simulation results of the novel filter when is excited with all higher-order modes (25 modes) 


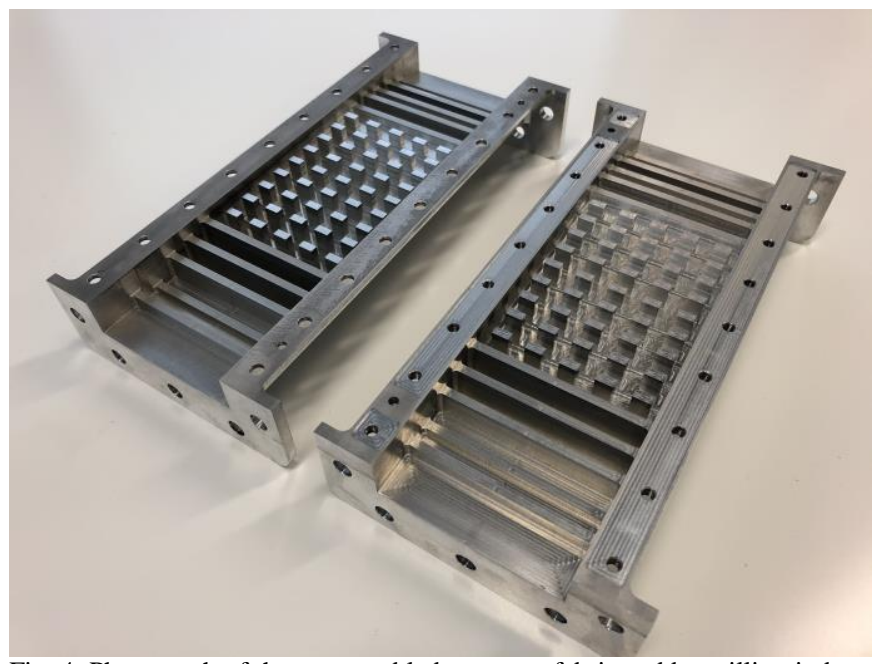

Fig. 4. Photograph of the unassembled structure fabricated by milling in bare aluminium

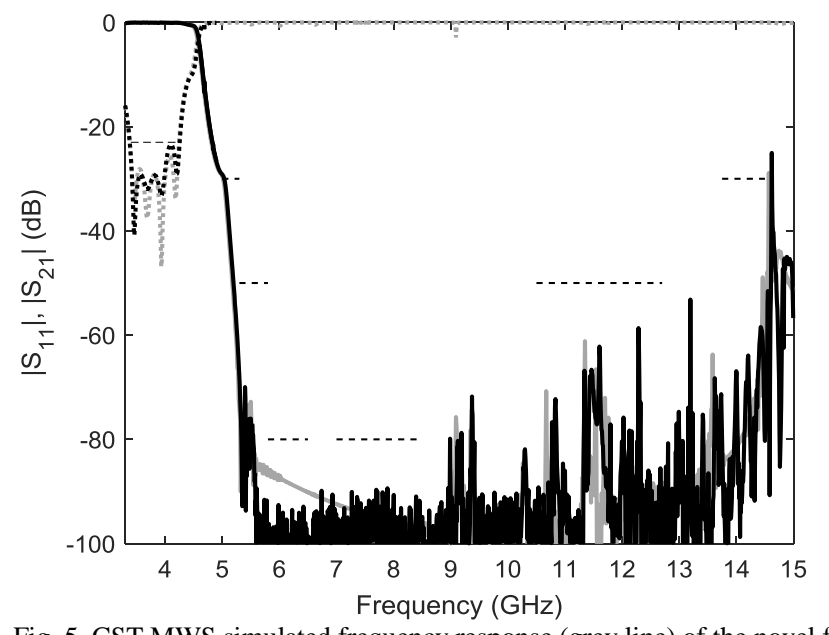

Fig. 5. CST MWS simulated frequency response (grey line) of the novel filter with rounded corners and measurements of the prototype (black line). $\left|S_{11}\right|$ in dotted line and $\left|S_{21}\right|$ in solid thick line. Frequency mask for return loss and outof-band rejection with dashed line.

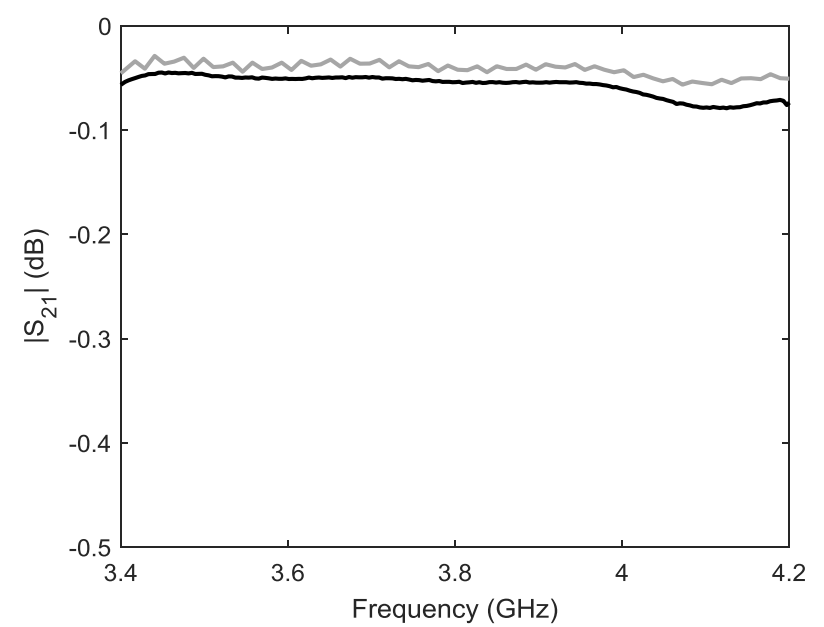

Fig. 6. Insertion loss detail. CST MWS simulated results in grey line (considering aluminium conductivity) and measurement results in black line. prediction considering 6 continuous wave carriers of $120 \mathrm{~W}$ each with $40 \mathrm{MHz}$ spacing has been performed using SPARK 3D. CST is used to calculate the electromagnetic fields at central frequency for each carrier which are used as inputs for the SPARK3D prediction. More than $10 \mathrm{~dB}$ margin with respect to the specifications was obtained, demonstrating excellent peak power handling operation. Peak power threshold could be further optimized by making use of the free parameters in the design method.

\section{CONCLUSION}

In this paper, a novel high-power filter for C-band satellite applications has been reported. With respect to other commercially available proposals, it achieves very reduced size, low losses and high-power handling, showing very promising results for the targeted application.

\section{ACKNOWLEDGMENT}

The authors would like to thank the European High RF Power Space Laboratory, European Space Agency and Val Space Consortium for contributing with its measuring installations - Laboratory co-funded by the European Regional Development Fund-A way of making Europe. This work was supported by the Spanish Ministerio de Ciencia, Innovación y Universidades - Agencia Estatal de Investigación under Project TE2017-85529-C3-2-R (AEI, FEDER-EU).

\section{REFERENCES}

[1] R. J. Cameron, C. M. Kudsia, and R. R. Mansour, Microwave Filters for Communication Systems: Fundamentals, Design and Applications. Hoboken, NJ: John Wiley \& Sons, 2007.

[2] G. L. Matthaei, L. Young, and E. M. T. Jones, Microwave Filters, Impedance-Matching Networks, and Coupling Structures. Norwood: Artech House, 1980.

[3] L. Young and B. M. Schiffman, "New and improved types of waffle-iron filters," in Proceedings of the Institution of Electrical Engineers, vol. 110, no. 7, pp. 1191-1198, Jul. 1963.

[4] E. Sharp, "A high-power wide-band waffle-iron filter," in IEEE Transactions on Microwave Theory and Techniques, vol. 11, no. 2, pp. 111-116, Mar. 1963.

[5] I. Arregui et al., "High-power low-pass harmonic waveguide filter with TEn0-mode suppression," in IEEE Microwave Wireless Components Letters, vol. 22, no. 7, pp. 339-341, Jul. 2012.

[6] F. Teberio et al., "Design procedure for new compact waffle-iron filters with transmission zeros," in IEEE Transactions on Microwave Theory and Techniques, vol. 66, no. 12, pp. 5614-5624, Dec. 2018.

[7] F. Teberio et al., "Low-loss compact Ku-band waveguide low-pass filter," IEEE MTT-S, Phoenix, May 2015.

[8] (2019) ESA-ARTES website. [Online]. Available: https://artes.esa.int/news/tesat-spacecom\%E2\%80\%99s-new-c-bandportfolio 